Itinéraires Itinéraires

Littérature, textes, cultures

2019-2 et $3 \mid 2019$

Corps masculins et nation : textes, images, représentations

\title{
Les corps des hommes, le corps de la nation
}

Introduction

Bodies of Men, Body of the Nation

Sergio Coto-Rivel, Cécile Fourrel de Frettes et Jennifer Houdiard

\section{OpenEdition}

\section{Journals}

Édition électronique

URL : http://journals.openedition.org/itineraires/7127

DOI : $10.4000 /$ itineraires. 7127

ISSN : 2427-920X

Éditeur

Pléiade

Référence électronique

Sergio Coto-Rivel, Cécile Fourrel de Frettes et Jennifer Houdiard, «Les corps des hommes, le corps de la nation », Itinéraires [En ligne], 2019-2 et 3 | 2019, mis en ligne le 11 décembre 2019, consulté le 24 septembre 2020. URL : http://journals.openedition.org/itineraires/7127 ; DOI : https://doi.org/ 10.4000/itineraires.7127

Ce document a été généré automatiquement le 24 septembre 2020.

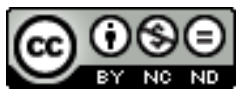

Itinéraires est mis à disposition selon les termes de la licence Creative Commons Attribution - Pas d'Utilisation Commerciale - Pas de Modification 4.0 International. 


\title{
Les corps des hommes, le corps de la nation
}

\author{
Introduction \\ Bodies of Men, Body of the Nation
}

Sergio Coto-Rivel, Cécile Fourrel de Frettes et Jennifer Houdiard

\begin{abstract}
«Le monde social construit le corps comme réalité sexuée et comme dépositaire de principes de vision et de division sexuants. Ce programme social de perception incorporé s'applique à toutes les choses du monde, et en premier lieu au corps

lui-même, dans sa réalité biologique [...]. »

Pierre Bourdieu, La Domination masculine.
\end{abstract}

1 Depuis une dizaine d'années les études sur le genre et les masculinités gagnent de la place dans le paysage académique français grâce aux nouvelles approches théoriques d'analyse culturelle venues particulièrement des pays anglophones (Connell 2014 et Butler 2006, entre autres), et ce, malgré l'opposition de plus en plus criante de certains groupes religieux et d'une partie de la société civile - La Manif pour Tous (LMPT), Civitas, Sens commun - qui dénoncent une manipulation idéologique de la part des universitaires dans le but de défaire le genre organisant les modes de fonctionnement sociaux et les rapports de domination.

Dans ce contexte de forte remise en cause des luttes pour l'égalité femmes-hommes et pour les droits des personnes LGBTIQ, ce numéro double d'Itinéraires entend mettre en avant des regards pluriels dans l'appréhension de ce que l'on place traditionnellement sous le terme, souvent utilisé au singulier, de "masculinité ». Afin d'apporter un point de vue critique sur nos sociétés contemporaines et de faire apparaître une réalité diverse, nous avons fait le choix de nous intéresser aux productions littéraires et iconographiques de différentes aires géographiques et linguistiques, tout en resserrant le propos sur ce qui nous a semblé essentiel dans le processus de construction du masculin : le rapport au corps et le lien avec la nation, ce lien identitaire n'étant pas sans incidence sur le corps des hommes. 


\section{Une perspective multiculturelle et pluriartistique}

3 Tout d'abord, la genèse du projet mérite d'être éclairée : le présent dossier est une sélection d'articles issus des communications prononcées lors du colloque «Corps masculins : représentations littéraires et iconographiques de la Nation", organisé par le Centre de recherche sur les identités, les nations et l'interculturalité (CRINI) de l'université de Nantes et le laboratoire Pléiade de l'université Paris 13, les 19 et 20 avril 2018. Il s'insère dans les perspectives inter-langues, interculturelles et interdisciplinaires de ces deux laboratoires et poursuit une réflexion amorcée il y a quelques années, lors de la journée d'études «Masculinités hispaniques : déviances et résistance dans la littérature hispanophone contemporaine ${ }^{1}$ ».

Cette première rencontre avait posé la question du genre et de ses expressions artistiques et culturelles. Elle avait mis en évidence, d'une part, le lien intime qui existe entre les représentations des masculinités et celles du corps, que celui-ci soit individuel ou collectif, physiologique ou symbolique. D'autre part, elle avait fait émerger la question centrale de l'identité, et particulièrement de l'identité nationale, dans les définitions du masculin: de la virilité de l'homme-soldat exaltée par les rhétoriques prétoriennes aux déconstructions les plus radicales de la masculinité traditionnelle, du roman nationaliste costaricain des premières années $\mathrm{du} \mathrm{xx}^{\mathrm{e}}$ siècle aux écrivain'e's queer qui publient depuis quelques décennies en Colombie, au Mexique ou en Espagne, nous avions vu s'esquisser, au fil des contributions, un portrait contrasté de masculinités hispaniques aussi multiples que mouvantes. De cette journée est née l'idée d'élargir la réflexion à d'autres aires culturelles et à des formes d'expression artistique non seulement textuelles mais aussi iconiques - le cinéma, par exemple - tout en interrogeant plus précisément l'articulation entre corps et nation.

5 Notre intérêt pour la création contemporaine et les cultures populaires, couplé à notre souhait de diffuser nos questionnements scientifiques hors des murs souvent intimidants de l'institution universitaire, nous a convaincuee's d'introduire le colloque "Corps masculins », par une performance du slameur, comédien et musicien D' de Kabal. Artiste résident de l'université Paris 13, où il intervient notamment auprès des étudiants, il a interprété sa conférence musicale «Masculin/Féminin » sur le campus nantais du Tertre le 16 mars 2018. Celle-ci s'inspire en partie de l'expérience de l'artiste, fondateur d'un « laboratoire de déconstruction et redéfinition du masculin par l'art et le sensible »: il est constitué d'ateliers, nés en 2015 à Canal 93 (Bobigny) et menés, depuis lors, dans divers lieux de la région parisienne, ainsi qu'à Kourou et Fortde-France ; ces ateliers réunissent des hommes volontaires dans des groupes de parole non mixtes, avec l'objectif de déconstruire les masculinités toxiques et d'imaginer un masculin libéré du carcan des injonctions virilistes. Cette manifestation, suivie par une discussion à laquelle un large public - universitaires, étudiante's et spectateur.ice's d'horizons divers - a pris part, nous a permis d'amener la problématique que nous avons placée au centre de notre démarche scientifique.

\section{Mythes et discours du masculin}

6 L'intérêt grandissant, non seulement dans les sciences humaines mais aussi dans les médias traditionnels, pour les différentes idées et représentations des masculinités, 
voire de la virilité, est de plus en plus évident: publication d'ouvrages théoriques, études de cas, essais historiques ${ }^{2}$ et philosophiques, documentaires, etc. Il suffit de prêter l'oreille aux débats qui se tiennent dans les émissions de radio ou de télévision pour se convaincre de cette préoccupation récurrente à propos du masculin et des traits qui lui seraient associés ${ }^{3}$, ou qui au contraire s'en éloigneraient. Bien sûr, cela fait écho à d'autres sujets polémiques tels que la place des femmes en politique, la discrimination en raison de la préférence sexuelle ou le mouvement \#MeToo. De la même manière, l'idée de masculinité toxique est dénoncée de plus en plus dans des productions culturelles populaires telles que la musique, comme c'est le cas de Stromae et Eddy de Pretto, pour ne citer que deux exemples francophones ${ }^{4}$ qui s'attaquent aux effets néfastes d'une certaine conception du masculin, comme la violence exercée sur les femmes pour le premier, et les clichés de la virilité pour le second.

7 Cette constatation nous renvoie inévitablement à une idée très souvent évoquée, celle de la crise de la masculinité : l'homme traditionnel est-il en crise et quelles en seraient les manifestations? Francis Dupuis-Déri (2018), consacre un ouvrage à cette question en la qualifiant, dès l'intitulé, de "mythe tenace». En effet, celui-ci réapparaît constamment dans nos sociétés, ce qui l'apparente plutôt à un discours :

Le discours de la crise de la masculinité s'inscrit le plus souvent dans une perspective très subjective, par exemple lorsque les hommes sentent que leur mère, leur conjointe ou leur ex-conjointe les dominent et lorsqu'ils sentent que la société est dominée par des femmes. Ces impressions et ces sentiments des hommes au sujet des femmes suffisent pour échafauder des grandes théories sans comparer ces abstractions - ces idées - avec la réalité. (Dupuis-Déri $2018: 38$ )

Selon Dupuis-Déri, le fait de se sentir menacé serait constitutif des masculinités patriarcales : cette impression ne serait donc pas due à une intervention extérieure visant à réduire l'influence et le pouvoir des hommes. En réalité, la virilité serait fragile presque par définition ${ }^{5}$ : ce ne serait qu'un idéal à atteindre, pour répondre à une série d'injonctions culturelles pouvant, de ce fait, être historicisées. En ce sens, la virilité ne recouvre pas toutes les masculinités possibles.

\section{Masculinités et virilité}

8 À l'instar d'études contemporaines sur la question, il convient de rappeler que ces deux termes ne sont pas synonymes. En effet, l'idée de «masculinités » au pluriel renvoie à toute une série de possibilités performatives des sujets mâles, tandis que la virilité possède une histoire particulière, celle de la domination: elle "désigne un idéal normatif, celui de l'excellence masculine. Si tout porteur d'un sexe masculin est un homme, tout homme n'est pas viril: seuls les meilleurs d'entre eux méritent ce qualificatif, aussi difficile à conquérir qu'à conserver" (Gazalé 2017: 198). Nous touchons là à un point fondamental dans la question du "devenir homme " ", susceptible de requérir un certain nombre d'étapes ou des rites de passages, dans les sociétés.

9 À ce propos, Mélanie Gourarier, dans une étude récente (2017), montre comment la construction du masculin passe par un discours de la «menace » : celle ressentie par les hommes vis-à-vis des femmes et des discours féministes. En particulier, elle s'intéresse aux manifestations d'un masculinisme récent qui réunit des hommes bien décidés à s'organiser pour stopper la montée du pouvoir féminin. Dans les stratégies prônées par ces masculinistes, le fait de séduire des femmes joue un rôle essentiel. La séduction 
participe, en effet, du maintien des liens de sociabilité des hommes entre eux, et leur permet de conserver leur domination. Une fois encore, la perception de la crise de la masculinité est un élément central pour comprendre le fonctionnement du système patriarcal :

C'est en effet parce que la masculinité est tenue pour menacée qu'il est possible de justifier sa défense et, par là même, de la renforcer. La crainte de son affaiblissement ne relève pas seulement du diagnostic ou de la nostalgie, elle a également pour fonction dynamique de travailler à la (re)production de la domination masculine. (Gourarier $2017: 40$ )

Une supposée masculinité en crise s'avère donc essentielle à son bon fonctionnement : elle a ainsi le droit d'être protégée. Par conséquent, elle serait une des stratégies de perpétuation de la virilité comme système de domination.

\section{Corps masculins et identité nationale}

Lorsque l'on évoque cette reproduction de la domination masculine, on peut difficilement faire abstraction de ses effets sur les corps des hommes. Cependant, ainsi que le rappelle Jean-Jacques Courtine, dans son Histoire $d u$ corps, il n'en a pas toujours été ainsi: "Le $\mathrm{xx}^{\mathrm{e}}$ siècle a inventé théoriquement le corps", déclare-t-il. Cette découverte - qui, selon lui, passe par plusieurs étapes de la psychanalyse à l'anthropologie, en passant par la phénoménologie, notamment - a permis «la prise en compte de l'image du corps dans la formation du sujet». Une dernière étape, fondamentale, se produit à partir des années 1960, au moment où le corps passe « du côté des catégories opprimées, marginalisées de la société » qui le brandissent pour protester contre l'ordre établi. Depuis lors, le corps ne peut plus être pensé en dehors des « marques de genre, de classe ou d'origine » qu'il porte (2006: 8-9).

11 De ce point de vue, que l'on adhère ou non à la pensée de Michel Foucault, la question du corps est liée à celle du pouvoir. Pour ce qui est du corps des hommes, il existe depuis longtemps des attributs associés à une certaine idée de la masculinité, censée protéger la nation ou la patrie. Ces deux derniers termes, genrés au féminin en français - et dans bien d'autres langues latines -, sont souvent incarnés par des femmes Marianne, Germania - qui les dotent d'un corps symbolique ${ }^{7}$. Cependant, traditionnellement, ce sont bien les hommes qui se portent garants de l'intégrité de la nation - ou de la patrie - dès que celle-ci se trouve en danger. Elle est en outre le lieu de la transmission d'un pouvoir qui est longtemps passé d'homme(s) à homme(s).

Dans Histoire de la virilité, Courtine (2011) accorde une place importante aux rapports entre la virilité et les instances de pouvoir au sein des États-nations; il se penche en particulier sur l'usage de la force, le contrôle et la défense de l'honneur, autant d'attitudes qui mettent en jeu le corps des hommes. Cependant, la relation entre les attributs virils et les caractéristiques de la nation, presque évidente dans le cas de l'armée, corps défendant de la nation, n'est ni immuable, ni univoque.

Le psychanalyste Michel Schneider (2002), par exemple, fait une distinction entre les tâches maternelles et paternelles de l'État pour dénoncer une dérive maternelle de ce dernier, ce qui montre encore un fort attachement, parfois réactionnaire, à l'idée que l'État s'entend en fonction d'une série de caractéristiques qui seraient proprement féminines ou masculines. D'où cette question binaire posée par Schneider : l'État doit-il être vu comme une mère nourricière ou comme un père protecteur? De fait cette 
interrogation émane d'une pensée qui ne s'est pas émancipée des présupposés de la société patriarcale, dans laquelle le terme "patrie " est indissociable de son origine étymologique latine, pater. Ainsi, alors que l'expression "mère patrie " correspond souvent à une construction identitaire qui renvoie au giron maternel et maternant, c'est bien la violence qui est mise en avant dans son pendant masculin viriliste.

Dès 1983, l'œuvre de référence Imagined Communities, de Benedict Anderson, avait apporté un nouvel éclairage sur les modes de fonctionnement en communauté et le processus de formation de l'imaginaire lié à la nation. Les origines de la conscience nationale et les représentations qui lui ont été associées s'avèrent inséparables des caractérisations du corps masculin. En effet, la virilité a été utilisée de manière très productive dans les descriptions idéales de la nation et de ses représentants (nous pensons par exemple à la relation entre le corps de l'homme idéal dans l'image de l'armée et celle-ci comme représentation de la nation tout entière).

Plus récemment, Tod W. Reeser a analysé que, dans le discours de la nation et de la masculinité, ces caractérisations sont "imaginées, limitées et démarquées » (2010: 175). Ce phénomène est visible tant dans les représentations viriles de l'armée que dans les traits féminins ou efféminés attribués à l'ennemi. Selon ce spécialiste, «la masculinité peut aider à faire apparaître la nationalité comme naturelle ». Ainsi, la naturalisation de la nation et celle du corps masculin s'insèrent au sein d'un seul et même processus discursif.

\section{Composition du dossier}

16 Ce processus discursif de construction identitaire est, évidemment, complexe et possède des caractéristiques propres aux aires culturelles dans lesquelles il a lieu. La diversité des productions ici étudiées - romans, récits de témoignage, pièces de théâtre, presse, films, bandes dessinées, traités de médecine, entre autres - entend en donner un aperçu, sans prétendre bien entendu à l'exhaustivité.

La série d'articles qui ouvre ce dossier explore, dans différents contextes, la construction d'une masculinité où la virilité du corps mâle est glorifiée. Les chants des soldats étudiés par Mélanie Texier en sont une manifestation criante : à la Légion étrangère, alors que le rapport à la nation se trouve en quelque sorte déplacé vers un autre collectif, la force guerrière du corps n'en est pas moins exaltée ; elle est d'ailleurs exclusivement masculine. Cet article centré sur la notion d'«incorporation langagière", se base sur un vaste corpus de chansons et d'entretiens avec des militaires, pour mettre en lumière la manière dont le soldat "fait corps " avec le collectif - la Légion -, mais aussi comment se manifeste la forte sexualisation du corps de chacun. La physionomie super-héroïque de Batman est, en quelque sorte, une représentation hyperbolique de ce corps masculin très sexualisé, érigé en protecteur d'une nation, dont la ville de Gotham est une allégorie. C'est dans cette perspective que Sophie Bonadè analyse, dans les comic books parus depuis les années 1980, le rapport du célèbre personnage à un espace urbain qui n'est pas sans évoquer quelque mégalopole étatsunienne. Dans El Conspirador. Autobiografía de un hombre público. Novela político-social de la Péruvienne Mercedes Cabello de Carbonera, le contexte de référence est plus explicite: la guerre du Pacifique (1879-1883) et la période immédiatement postérieure, où les caudillos ${ }^{8}$ se succédèrent à la présidence. L'œuvre n'en restitue pas moins des mémoires fictives, celle du leader Jorge Bello, dont l'idéal viril et autoritaire 
va bientôt vaciller au profit d'un modèle alternatif - plus respectueux des intérêts collectifs de la nation - qui se traduit, selon Sarah Porcheron, par une « féminisation du caudillo".

18 Le recours à la féminisation des corps masculins est un procédé polysémique qui peut répondre à des objectifs divers, ainsi que le montre le deuxième volet de ce dossier. Tout d'abord, Jean-Christophe Corrado se centre, dans son étude, sur une dimension symbolique de la féminisation de la nation propre au contexte franco-allemand décrit par Jean Genet dans Pompes funèbres : selon lui, les oppositions de genre servent, dans cette œuvre, à décrire les relations entre personnages masculins, allemands et français, non pas en termes d'opposition, mais sur le mode d'un couple homme-femme. Il est néanmoins plus fréquent que la féminisation des corps masculins soit le signe de leur décadence. C'est notamment le cas dans deux récits de la Première Guerre mondiale étudiés par Maria Chiara Gnocchi - Le Feu de Henri Barbusse (1916) et La Peur de Gabriel Chevallier (1930) - où le procédé renvoie à une dégradation de l'idéal patriotique : le conflit ne fut pas la grande épopée virile dont rêvaient certains; au contraire, l'expérience des tranchées aurait dévirilisé les "poilus", qui vinrent former une catégorie à part, en marge des autres hommes. La féminisation permet en outre de rabaisser l'autre, pour mieux le dominer. Telle fut pendant longtemps la position dans laquelle on plaça l'homme africain, décrit par la littérature médicale française du XIX siècle et de la première moitié $d u x^{e}$ comme un être efféminé, physiquement et intellectuellement, ce qui légitimait la colonisation de ses terres par des hommes blancs, considérés comme plus virils et donc plus puissants. Delphine Peiretti-Courtis note toutefois que cette représentation a coexisté avec une autre vision, tout à fait opposée, celle de l'homme noir hypervirilisé, qui devint même un exemple pour l'homme blanc.

19 Quoi qu'il en soit, il n'est pas rare que le féminin soit associé à des valeurs négatives et suscite méfiance ou rejet. D’ailleurs, ce dénigrement fonctionne généralement en opposition au masculin qui s'en trouve alors valorisé. Aussi la troisième partie du numéro se centre-t-elle sur plusieurs types de résistances, que l'on pourrait qualifier de masculinistes. Chez Montherlant, par exemple, la féminité est présentée comme une gangrène qui met en danger non seulement le corps du héros des Jeunes filles (1936-1939), mais aussi celui d'une nation menacée d'émasculation, même si, selon l'analyse de Pierre Damamme, cette horreur du féminin n'est peut-être pas à prendre au premier degré. En revanche, c'est bien contre le féminin que se construit l'identité nationale mise en avant par la revue espagnole Nuestro Cinema, au début des années 1930. Évelyne Coutel montre comment, face à la montée du pouvoir des femmes, cette publication défend une masculinité hégémonique et normative qui rejette les représentations hollywoodiennes, jugées déviantes, pour prendre exemple sur le modèle viriliste offert par le cinéma russe. Il apparaît donc que, contrairement aux idées reçues, idéologie libertaire et féminisme ne vont pas nécessairement de pair. Dans son article sur la révolution sandiniste au Nicaragua dans les années 1980, Viria Delgadillo rejoint cette analyse. Les textes et les images étudiés révèlent une logique masculiniste et hétérosexiste, en contradiction avec le projet de société égalitaire mis en avant par le discours révolutionnaire.

Même si ce sont les hommes qui conduisent les nations, les normes qui définissent cette masculinité hégémonique ne sont pas immuables et sont sujettes à caution. Encore aujourd'hui, le cinéma français reconduit des représentations traditionnelles du 
pouvoir, incarné par la figure du père, tandis que la femme, elle, n'y a jamais accès. Bien que les films récents analysés par Robin Hopquin ont indéniablement recours au paternalisme, ils révèlent également un malaise face à un idéal tantôt inaccessible, tantôt insatisfaisant. Inversement, dans l'Amérique de la seconde moitié des années 1930, il est des acteurs, comme Spencer Tracy, qui remportent une vaste adhésion auprès du public. Érigée en icône de la nation, malgré ses contradictions, cette star témoigne d'une reconfiguration de la masculinité hégémonique dans le contexte propre aux années qui suivirent la Grande Dépression, ce qui permet à Jules Sandeau de conclure à une certaine plasticité du modèle de masculinité hégémonique aux ÉtatsUnis. Le contexte politique est également déterminant dans l'évolution des personnages masculins du dramaturge irlandais Owen McCafferty, étudié par Brigitte Bastiat. Dans quatre pièces écrites et jouées entre 1994 et 2017, c'est la violence symbolique et physique de l'État qui amène les hommes à reconstruire leur masculinité, ce qui n'est pas sans remettre en question un modèle national imposé.

21 Le dernier volet de ce dossier s'intéresse aux masculinités transgressives qui conduisent à redéfinir l'« être homme », dans des productions interrogeant l'identité nationale au prisme de l'homosexualité. La première étude, menée par Emmanuel Le Vagueresse, s'arrête sur le cinéma à la fois militant et commercial d'Eloy de la Iglesia. Dans la seconde moitié des années 1970, alors que la transition démocratique débute à peine en Espagne, il est l'un des premiers à montrer des corps masculins éveillant un désir sexuel libéré de l'hétérocentrisme : non seulement son film de 1977 intitulé Los placeres ocultos (Les plaisirs cachés) porte la "question gay » sur le devant de la scène, mais il renouvelle, de plus, la représentation de l'homosexuel, loin des clichés, à un moment où la nation espagnole se redéfinit dans sa marche vers la démocratie. De même, The Swimming Pool Library (1988) d'Alan Hollinghurst fit date en Angleterre. Quoique d'autres, avant lui, aient évoqué l'homosexualité masculine, l'angle choisi par l'auteur, dans ce premier roman, était tout à fait novateur : pratiques et habitudes de la communauté gay y sont évoquées sans tabous. Surtout, ainsi que le démontre Georges Letissier dans son article, la grande originalité de cet écrivain réside dans le choix de repenser le lien entre masculinité et nation à partir de l'homosexualité, généralement reléguée en marge du récit historique. Ainsi, dans L'Enfant de l'étranger (2011), il renverse le mythe du poète-combattant, héros de la Grande Guerre, pour proposer une autre mémoire de la nation, où une place est faite à l'intime masculin. La perspective d'Hollinghurst, non dépourvue d'essentialisme, distingue son œuvre de la pensée et de l'art queer. C'est en revanche sur ce concept que se penche Juliette Ledru dans son article consacré aux représentations de la masculinité dans la communauté chinoise aux États-Unis. Son étude porte sur deux films, qui évoquent les difficultés des SinoAméricains à s'identifier aux modèles de représentation culturels qui leur sont proposés : Garçon d'honneur (1993) d'Ang Lee - dont le personnage principal est à la fois ouvertement gay et en couple au regard de ses amis américains, et pour autant « dans le placard" et prétendument hétérosexuel pour ses parents et membres de la communauté chinoise de New York - et Red Doors (2005) de Georgia Lee, où apparaît la figure d'un patriarche suicidaire. L'analyse permet de mettre en lumière les enjeux de cette masculinité sino-américaine, qui se présente comme un lieu en tension, de négociation et de résistance, face aux modèles dominants. 


\section{BIBLIOGRAPHIE}

Aeschiman, Éric, 2002, «Je reproche à l'État d'être une mauvaise mère » (Entretien avec Michel Schneider), Libération, 22 juin 2002, [En ligne], https://www.liberation.fr/week-end/2002/06/22/ je-reproche-a-l-etat-d-etre-une-mauvaise-mere_408028, consulté le 2 décembre 2019.

Bourdieu, Pierre, [1998] 2002, La Domination masculine, Paris, Seuil.

Butler, Judith, 2006, Défaire le genre, Paris, Éditions Amsterdam.

Connell, Raewyn, 2014, Masculinités. Enjeux sociaux de l'hégémonie, Paris, Éditions Amsterdam.

Coto-Rivel, Sergio, Fourrel de Frettes, Cécile et Houdiard, Jennifer (dir.), 2017, GRAAT On-Line, $\mathrm{n}^{\circ}$ 20, novembre 2017, « Masculinités hispaniques : déviances et résistances dans la littérature hispanophone contemporaine », [En ligne], http://www.graat.fr/backissuemasculinity.html, consulté le 2 décembre 2019.

Courtine, Jean-Jacques (dir.), 2006, Histoire du corps 3. Les mutations du regard. Le XX $x^{e}$ siècle, Paris, Seuil.

Courtine, Jean-Jacques, Corbin, Alain et Vigarello, Georges (dir.), 2011, Histoire de la virilité 3. La virilité en crise. Le $\mathrm{XX}^{e}-\mathrm{XXI}^{e}$ siècles, Paris, Seuil.

Dupuis-Déri, Francis, 2018, La Crise de la masculinité. Autopsie d'un mythe tenace, Montréal, Les Éditions du remue-ménage.

Donapetry, María, 2006, Imagi/nación: la feminización de la nación en el cine español y latinoamericano, Madrid, Ed. Fundamentos.

Gazalé, Olivia, 2017, Le Mythe de la virilité. Un piège pour les deux sexes, Paris, Robert Laffont. Gourarier, Mélanie, 2017, Alpha mâle. Séduire les femmes pour s'apprécier entre hommes, Paris, Seuil. Jablonka, Ivan, 2019, Des hommes justes. Du patriarcat aux nouvelles masculinités, Paris, Seuil. Kosofsky Sedgwick, Eve, 1990, Epistemology of the closet, Berkeley, University of California Press. Reeser, Tod W., 2010, Masculinities in Theory, Oxford, Wiley-Blackwell.

\section{NOTES}

1. Cette journée, organisée par les laboratoires Pléiade et CRINI, s'est tenue à Nantes le 14 octobre 2016. Elle a donné lieu à une publication dans une revue en ligne consacrée aux cultural studies (Coto-Rivel, Fourrel de Frettes et Houdiard 2017).

2. Citons, à titre d'exemple, une des dernières parutions en date, l'ouvrage d'Ivan Jablonka (2019).

3. Le cas en France du polémiste Éric Zemmour, ses commentaires à la télévision et à la radio, ainsi que son livre Le Premier sexe (2006), illustrent ce besoin de mettre en avant la question masculine pour, selon Zemmour, défendre les hommes face aux féministes et leur prise de pouvoir.

4. Les chansons Tous les mêmes de Stromae et Kid de Eddy de Pretto. 
5. Par exemple, à cause de la notion de "panique homosexuelle » développée par Eve Kosofsky Sedgwick dans l'ouvrage déjà classique, Epistemology of the closet (1990).

6. Si l'on reprend la fameuse formule de Simone de Beauvoir.

7. Voir par exemple, à ce propos, l'étude de María Donapetry (2006).

8. Chef militaire qui a pris le pouvoir (Trésor de la langue française).

\section{AUTEURS}

\section{SERGIO COTO-RIVEL}

Université de Nantes, CRINI (EA 1162)

CÉCILE FOURREL DE FRETTES

Université Paris 13, Pléiade (EA 7338)

JENNIFER HOUDIARD

Université de Nantes, CRINI (EA 1162) 